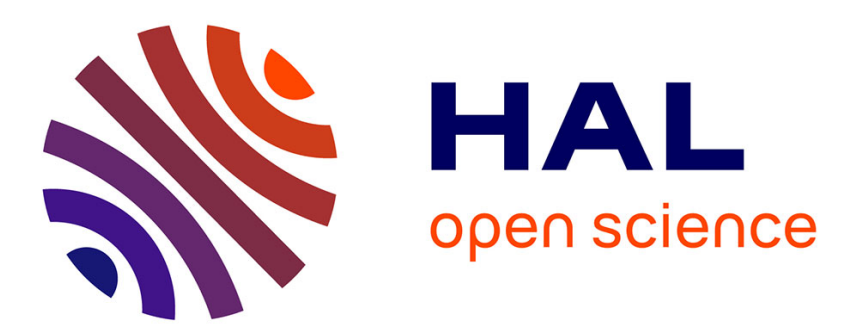

\title{
La vulnérabilité alimentaire des Sahéliens : concepts, échelles et enseignements d'une recherche de terrain
}

Pierre Janin

\section{To cite this version:}

Pierre Janin. La vulnérabilité alimentaire des Sahéliens: concepts, échelles et enseignements d'une recherche de terrain. Espace Géographique, 2006, 4, pp.355-366. ird-00275237

\section{HAL Id: ird-00275237 \\ https://hal.ird.fr/ird-00275237}

Submitted on 22 Apr 2008

HAL is a multi-disciplinary open access archive for the deposit and dissemination of scientific research documents, whether they are published or not. The documents may come from teaching and research institutions in France or abroad, or from public or private research centers.
L'archive ouverte pluridisciplinaire HAL, est destinée au dépôt et à la diffusion de documents scientifiques de niveau recherche, publiés ou non, émanant des établissements d'enseignement et de recherche français ou étrangers, des laboratoires publics ou privés. 


\title{
La vulnérabilité alimentaire des Sahéliens : concepts, échelles et enseignements d'une recherche de terrain
}

Article paru dans L'Espace Géographique, n 4/2006, pp. 355-366.

\author{
Pierre JANIN \\ IRD - UR 106 « Nutrition, alimentation, sociétés » \\ Iedes/Université de Paris I \\ 45 bis, avenue de la Belle Gabrielle \\ 94736 Nogent sur Marne cedex \\ tél. : 0143947237 \\ fax. : 0143947246 \\ mél. : Pierre.Janin@ird.fr
}

RÉSUMÉ. - L'étude géographique de la vulnérabilité alimentaire en milieu rural passe presque toujours par une description du « milieu » tout en cherchant à mieux prendre en compte la variabilité temporelle et spatiale des situations, à différentes échelles. La recherche, menée au Burkina Faso, montre que le niveau de risque alimentaire, évalué d'après les contraintes environnementales, est loin de correspondre à la vulnérabilité réelle des ménages. Ceci tend à conforter l'idée que la définition et la collecte d'indicateurs pertinents passent par une connaissance approfondie des modes de régulation sociale et des formes de gestion du risque d'insécurité alimentaire.

VULNÉRABILITÉ, RISQUE, SOUDURE ALIMENTAIRE, INDICATEURS, BURKINA FASO

\begin{abstract}
Food vulnerability of Sahelian people: concepts, scales and lessons of a field research program. - The geographical study of food vulnerability in the Sahel region of West African almost always begins with a descriptive analysis of the environment. In light of the variability and uncertainty of risk situations, greater account is gradually being taken of their temporal and spatial dimensions on various scales. However, research conducted in Burkina Faso shows that food risks levels assessed through environmental factors do not reflect the actual vulnerability of households. This research supports the argument that the definition and collection of relevant indicators require a thorough knowledge of social control and local management of food insecurity risk.
\end{abstract}


Le cadre théorique d'analyse de la vulnérabilité alimentaire paraît fermement établi si l'on en juge la très large diffusion de la définition qui l'inspire (Hamelin et Habicht 1998 ; Dilley et Boudreau 2001; Delor et Hubert 2000). Cette dernière s'organise autour des notions d'exposition, de sensibilité et de réactivité (encore parfois appelée résilience) différenciées face à des risques (d'insécurité alimentaire et de malnutrition) par la mobilisation de ressources physiologiques, économiques, sociales ou éco-géographiques (Courade et al. 2001, Banque Mondiale 2001). La vulnérabilité doit donc être considérée comme la capacité différenciée d'un individu ou d'une famille à endurer des stress, de toute nature, à les contourner éventuellement et à réagir en mobilisant des ressources matérielles, sociales ou symboliques, à court comme à plus long terme. Elle peut être transitoire ou posséder un dimension plus structurelle.



Un «système » ou un milieu naturel pourra être considéré comme vulnérable mais le concept s'applique plus directement à des acteurs individuels ou collectifs dans notre cas d'étude. La vulnérabilité se décline ainsi à différentes échelles spatiales ou statistiques, à l'aide d'une batterie d'indicateurs conjoncturels ou structurels susceptibles d'être modélisés à des fins comparatives. Et pourtant, les expériences de recherche englobante autour de la question du risque et de la vulnérabilité alimentaire, intégrant la diversité des acteurs, les temporalités d'action ou les transferts d'échelles spatiales semblent à la fois encore peu nombreuses et difficiles à mettre en œuvre.

Plusieurs raisons peuvent, tour à tour, être invoquées : la diversité des champs thématiques concernés, à la fois distincts et solidaires ; le décalage entre les cadres conceptuels, les perspectives méthodologiques et les enseignements pour l'action, parfois contradictoires, qui en découlent; la perméabilité aux discours non scientifiques (idéologiques et technocratiques) ou encore la variabilité des contextes locaux, ruraux et urbains (Raynaut et al. 1998). De fait, la mesure réelle de la vulnérabilité alimentaire semble vouloir échapper à toute analyse strictement disciplinaire, statistique ou normative.

C'est pourquoi, il convient d'adopter une approche assez large - des facteurs de production alimentaire, aux modes marchands et non-marchands de l'accessibilité en passant par les formes complexes de gestion et de redistribution des denrées - reconsidérant à la fois le caractère donné et construit du risque et les réponses sociales ou techniques, plus ou moins efficientes, qui en découlent. Une approche globale ne peut également laisser en friche l'ensemble des perceptions (Briand 2004), des normes intériorisés et des dispositifs hiérarchiques (le « contrôle social » ou les «modes de régulation ») susceptibles d'expliquer et d'orienter les décisions à court comme à plus long terme. Elle doit, enfin restituer au temps, qui se déroule plus ou moins lentement, la place qui est la sienne. Si l'on excepte les contextes de crise 
politique ou climatique aiguë, la vulnérabilité résulte donc d'une combinaison de facteurs parfois difficiles à mesurer, à hiérarchiser et à relier.

C'est une démarche construite pas à pas, associant choix inductifs et reconceptualisation déductive, "vérité terrain » et « lecture des risques », qui a été tentée dans l'Est du Burkina Faso $^{1}$. Elle a donné naissance à un ensemble coordonné d'enquêtes, répétées et emboîtées, dont nous ne présenterons que la composante géographique. Ce texte revient aussi sur les différentes dimensions spatio-temporelles de la vulnérabilité apparente et réelle ${ }^{2}$ afin d'identifier certains indicateurs instantanés dans l'optique d'une intervention alimentaire ou nutritionnelle éventuelle.

\section{Les approches institutionnelles}

Depuis le début des années 1990, la vulnérabilité alimentaire en milieu rural africain a été abordée sous deux angles :

- à une échelle administrative et statistique agrégée, confrontant seuils statistiques de couverture et de satisfaction des besoins (ration en kg/équivalent-adulte/an et en équivalent kilocalories) avec d'autres indicateurs avancés (prix sur les marchés, prévision extrapolée de récoltes) que des systèmes d'information s'efforcent de réactualiser afin de tenter de prévenir des crises manifestes. Cette "mesure » spatialisée des déficits alimentaires - s'appuyant sur une lecture agro-météorologique des risques à la fois conjoncturelle et structurelle - est de longue date privilégiée par les institutions telles que l'Organisation des Nations Unies pour l'Alimentation et l'Agriculture (Blein et al. 2001), le Comité Interétatique de Lutte contre la Sécheresse au Sahel (CILSS), le Centre régional de formation et d'application en agrométéorologie et hydrologie opérationnelle (AGHRYMET) (http://www.ibimet.cnr.it/Case/ap3a/site ap3a/produits.htm) ou le Famine Early Warning System (FEWS), à défaut d'être la plus efficace, compte tenu du caractère fragmentaire de l'information, du maillage spatial disponible et de la place minime accordée au comportement socio-économique des acteurs ou aux dynamiques d'accès aux ressources. C'est néanmoins l'approche encore souvent mise en œuvre pour améliorer la fluidité des échanges transfrontaliers sous-régionaux ou mobiliser d'éventuels donateurs d'aide.

- à une échelle à la fois macro- et micro- au fur et à mesure que les politiques agricoles et alimentaires mettaient davantage en avant le marché pour faire face aux situations d'insécurité alimentaire, même si leur gestion quotidienne incombe aux familles. D'où le soutien à une multitude d'actions très ciblées (activités génératrices de revenu, protection des sols), destinées à certains groupes, considérés a priori comme "porteurs » (femmes) ou vulnérables (migrants) dans les Cadres Stratégiques de Lutte contre la Pauvreté (CSLP) et des Conseils Nationaux de Sécurité Alimentaire (CNSA). Cette approche n'est cependant pas exempte de biais puisque les dynamiques sociales en œuvre (individuation, régulation) semblent quelque peu délaissées au profit de la collecte rapide d'indicateurs socio-économiques selon des protocoles bien établis, comparables d'un pays à l'autre.

A l'heure actuelle, la tendance est la généralisation d'une démarche mixte, combinant des données conjoncturelles et structurelles comme dans les travaux de l'International Food Policy

\footnotetext{
${ }^{1}$ Les enquêtes ont été réalisées, dans le cadre du programme « Vulnérabilité alimentaire et sécurité nutritionnelle » de l'UR 106 de l'IRD, initié et coordonné entre 2001 et 2004 par Georges Courade, directeur de Recherches. Cette recherche géographique de terrain a été menée, par G. Courade, P. Janin et F. de C. Ouedraogo, auprès d'un échantillon aléatoire commun de trente villages, représentant 615 ménages ruraux dans une province sahélosoudanienne de la partie orientale du Burkina Faso (la Gnagna). D'autres enquêtes ont parallèlement été réalisées par des chercheurs en nutrition publique.

${ }^{2}$ Espace résidentiel regroupant plusieurs cases en banco (terre), entourées par un mur d'enceinte, autour d'une cour centrale et constituant un système familial multipolaire, associant plusieurs centres de production, de consommation et de décision ayant des relations complexes. Elle a été appelée Unité Collective d'Habitat (UCH).
} 
Research Institute (2003; Heidhues et al. 2004) et du Programme Alimentaire Mondial (PAM 2002). Elle est essentiellement conduite à l'échelle des communautés villageoises et des familles, à partir des travaux théoriques d'A. Sen sur les inégales capacités et droits, en collectant un ensemble d'indicateurs économiques, géographiques et sociaux, auprès de "groupes-cibles", plus ou moins vulnérables, afin de mieux prendre en compte les dynamiques en œuvre.

Elle a donné naissance à un schéma conceptuel d'analyse de la vulnérabilité plus abouti qui rend presque caduc les modèles plus anciens. Grâce à l'application d'un protocole strict d'enquête, elle permet de calculer un score de vulnérabilité, de modéliser certaines situations ainsi que les comportements tout en autorisant les comparaisons régionales ou nationales (PAM 2001). Qu'elles résultent de la recherche ou d'institutions internationales, ces approches font désormais ce pari : établir des diagnostics pertinents (démasquer l'insécurité transitoire masquée (Janin 2001) ou la faim « sociale » cachée), allant jusqu'à prendre en compte les écarts (géographiques, temporels) et les inégalités sociales et économiques (Jaspers 1999).

\section{Les points critiques}

Pour étudier la vulnérabilité alimentaire, notre choix s'est porté sur un espace de transition sahélo-soudanien, de colonisation agropastorale (regroupant des communautés peule, mossi et gourmantché), caractérisé par la récurrence des épisodes de déficit alimentaire (Chastanet 1991, Gado 1993).

Nous avons d'abord cherché à approfondir nos connaissances sur ce milieu biogéographique et ces sociétés respectives. Ont ainsi été collectées, à Ouagadougou et dans la Gnagna, un ensemble de données secondaires relatives aux incertitudes bioclimatiques, à la dégradation des potentialités environnementales, à l'évolution des systèmes de production, aux structures démographiques ou encore au fonctionnement du système social.

Toutefois, nous nous sommes rapidement heurtés à deux problèmes majeurs : le caractère généraliste ou localisé des informations disponibles sur les « déterminants » de la vulnérabilité (sur les potentialités ou le système foncier par exemple), le manque de données chiffrées actualisées fiables et représentatives permettant de caractériser les situations alimentaires à l'échelle locale (surplus ou manque).

C'est pourquoi, nous avons cherché à les affiner par des observations ponctuelles de terrain. Ainsi différentes missions ont servi à tester, puis valider les hypothèses générales, l'approche conceptuelle et les méthodes d'enquête.

\section{Encart 2:}

Temps descriptif, plus ou moins immédiat, lié à la saisonnalité des récoltes, à l'amenuisement des réserves, à la dégradation des termes de l'échange et aux besoins physiologiques de la consommation.

Temps stratégique de la gestion combinée des ressources disponibles (bétail, céréales, revenus) et des modes d'accès physiques et économiques au marché accordant aux anticipations de décision et aux perceptions de risques une place plus grande.

Temps systémique enfin, de la régulation pérenne de l'accès et de la redistribution des ressources entre groupes d'acteurs individuels et collectifs, aux intérêts, logiques et pratiques composites, intervenant au sein du système alimentaire. 
Par exemple, si la soudure alimentaire ${ }^{3}$ était bien un phénomène récurrent, elle variait fortement selon l'échelle spatiale ou temporelle d'analyse (Reardon et Matlon 1989) alors même que les conditions "objectives" (de production) restaient sensiblement les mêmes. D’où l'idée que le temps (Gallais 1984 ; Marchal 1990 ; Dupré et al. 1999 ; Moseley 2001) - et ses différentes dimensions (cf. encart ci-dessus) - étaient une variable-clé de l'analyse (Janin 2004). Dans cet espace, la question de l'élevage est également vite apparue comme centrale en période de soudure comme dans la durabilité des systèmes ${ }^{4}$ (Turner 2002), alors même qu'elle est parfois négligée dans les actions de développement. D'où l'effort supplémentaire consenti à l'étude des pratiques d'embouche ou d'élevage pastoral. Par ailleurs, depuis la libéralisation, le marché occupe une place au moins aussi décisive que le stockage physique ou «l'épargne sur pied » (Makki et al. 2001) dans les situations localisées d'insécurité alimentaire, tant par le volume des approvisionnements à l'échelle régionale que par a part des achats familiaux en cas de déficit (Courade 1998; Janin 2006). Enfin, l'estimation approchée des disponibilités céréalières, monétaires ou patrimoniales et de leurs modes de gestion devait être à la base de la recherche en raison des inégalités qu'elle révèle. Ce qui nécessitait une collecte systématique de données jusqu'à l'échelle individuelle.

Figure 1 : Approche conceptuelle de la vulnérabilité alimentaire dans la Gnagna

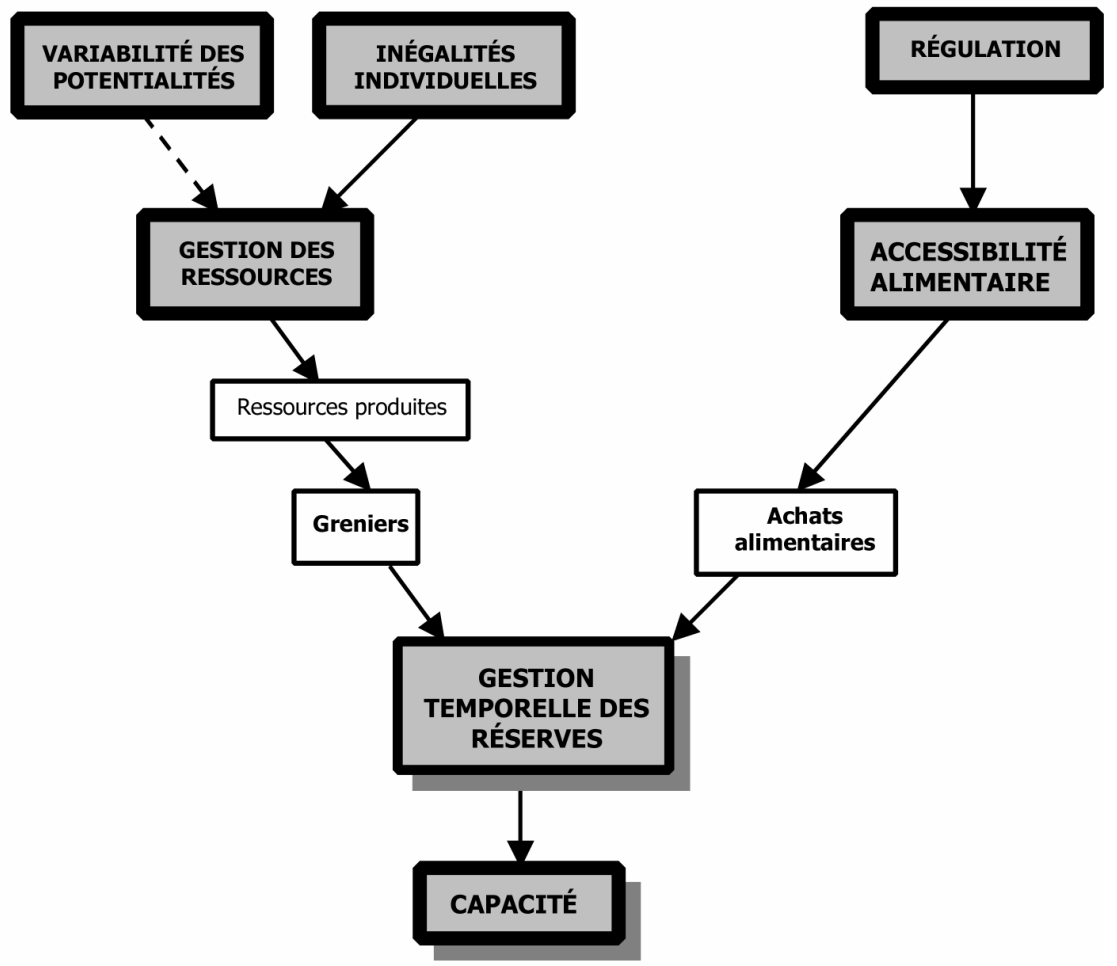

D'un point de vue conceptuel, nous avons préféré nous dégager des causalités liant $a$ priori sécheresse, pauvreté et insécurité alimentaire ou mettant en avant leur caractère surdéterminant (Burton 1997), même si l'environnement bio-climatique et géographique constitue un facteur aggravant de la vulnérabilité (Ribot et al. 1996). De fait, nous ne cherchions pas tant à caractériser des situations de «faim réelle ou cachée » ou de grande précarité qu'à mettre en

\footnotetext{
${ }^{3}$ Désigne l'intervalle entre la fin des réserves disponibles produites sur l'exploitation et la prochaine récolte céréalière et le déficit entre besoins alimentaires et disponibilités réelles.

${ }^{4}$ Reproduction socio-économique, association agriculture-élevage, restitution de la fertilité, complémentarité éleveurs-agriculteurs.
} 
évidence les interactions dynamiques entre risques (certains variables et aléatoires, d'autres récurrents), prise de décision plus ou moins anticipée et efficacité des réponses sociales ou techniques apportées en période de déficit alimentaire, c'est-à-dire le construit social de la vulnérabilité alimentaire. A tel point qu'il nous a semblé que les crises avérées (1913-1914, 19311932, 1973-1974, 1984-1985), au sens où l'entendent les décideurs, et compte tenu du volume important de l'aide mobilisée, n'avaient pas nécessairement des effets négatifs plus marqués que la succession de déficits annualisés, certes moins durables et moins sévères, mais foncièrement pernicieux à moyen terme parce qu'ils restaient « invisibles » (Bebbington 1999). Tout le travail de terrain a donc été calé sur l'année alimentaire des ménages qui débute avec le temps des récoltes (de la mi-octobre à la fin novembre) pour s'achever en fin d'hivernage de la l'année civile suivante. Ce qui nous a conduit à reconstituer patiemment, à l'aide de chronogrammes mensualisés, l'ensemble des événements passés ayant une incidence sur la situation alimentaire du ménage, avec une attention toute particulière pour la période de la soudure.

Par conséquent, trois thèmes corrélés ont été privilégiés (cf. figure 1) : l'accès aux facteurs de ressources et la valorisation des potentialités en ressources (Courade 2001), la gestion temporelle des ressources globales, les effets des modes de régulation lointains (État, aide internationale, marché). De manière plus précise, l'accessibilité géographique et économique à une ration alimentaire adaptée et choisie, comme l'autoconsommation ou les termes de l'échange bétail/céréales ont constitué les points critiques de ce type d'étude au même titre que la gestion temporelle des stocks, la redistribution éventuelle de vivres ou des revenus. En fonction de quoi, plusieurs «lieux » d'étude se sont rapidement imposés : la parcelle de culture, le grenier, le troupeau, la popote, le marché. En effet, chacun renvoie à un des aspects de la définition actualisée du concept de sécurité alimentaire : l'offre et les disponibilités dans le cas de la parcelle de culture et du grenier, l'accessibilité économique pour le troupeau et le marché, la stabilité en ce qui concerne la préparation et la redistribution alimentaire.

\section{Les différentes échelles et mesures}

Toute la difficulté a, ensuite, consisté à transcrire la problématique générale de la vulnérabilité en questions de recherche pertinentes avant de les reformuler, de manière plus explicite, en éléments pratiques d'enquête nécessitant la mise au point d'outils méthodologiques différenciés.

Pour ce faire, on a d'abord dressé une liste relativement exhaustive d'indicateurs à collecter à différentes échelles - régionale, villageoise, familiale et individuelle. Une sélection a, par la suite, été effectuée pour en conserver les variables les plus pertinentes et les plus accessibles.

Le dispositif d'enquête a été progressivement amendé en fonction du contexte scientifique : approche conceptuelle retenue, échelles spatio-temporelles pertinentes, contraintes logistiques et financières, exigences multiples de formation. Enfin différentes ébauches de questionnaire ont été réalisées avant d'être testées en « milieu réel ».

Nous sommes toutefois partis de l'idée, classique en géographie, d'élaborer un diagnostic territorial des handicaps, des contraintes, des potentialités résiduelles (hydrauliques, ligneuses, culturales,...) et des ressources mobilisables (aménagements et infrastructures) par les individus et les familles, à l'échelle de ce «petit » espace de $8400 \mathrm{~km}^{2}$. Il s'agissait donc d'appréhender la dimension la plus apparente de la vulnérabilité alimentaire, de ses déterminants géographiques premiers (Adger 1999, Moseley et Logan 2001, Ouedraogo 2001). Pour ce faire, ont été associées les données secondaires collectées et les déclarations des personnes enquêtées dans les 276 villages de la province. Cette première étape a donné lieu à l'établissement de cartes régionales pour chaque indicateur identifié.

Puis afin de mettre en exergue la nature des relations homme-milieu, certains indicateurs villageois ont ultérieurement été regroupés autour des thématiques géographiques des 
risques bio-climatiques, de la dégradation des ressources, de la soudure et de l'enclavement. Cette deuxième étape a permis d'aboutir à une caractérisation des inégalités spatiales à l'échelle provinciale. Quatre strates géographiques en résultent en fonction de l'intensité du phénomène observé (cf. figure 2). Elles donnent une certaine "image » de la province qui cadre assez bien avec les perceptions les plus communes des opérateurs de développement et des chercheurs eux-mêmes. C'est ce que nous avons appelé, pour les différencier du risque systémique (cf. introduction), les « risques apparents ».

Figure 2 : Niveaux de risque apparent
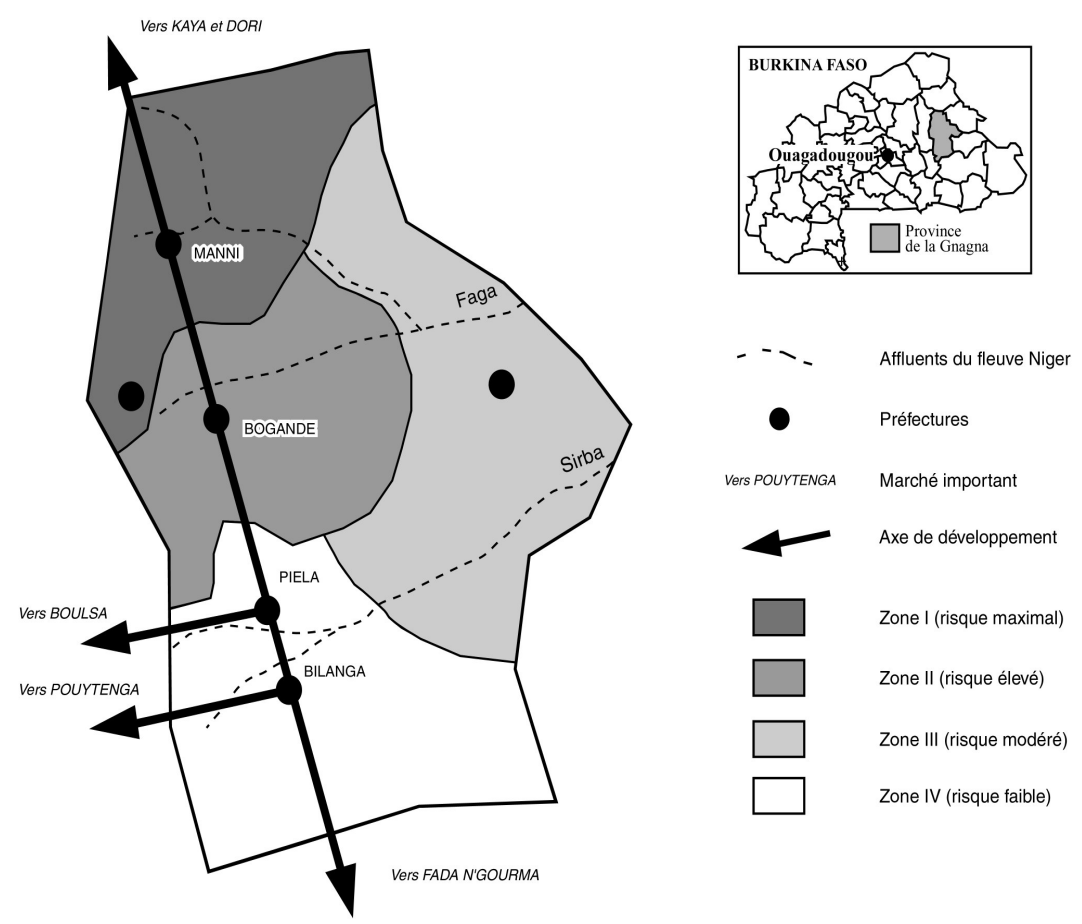

\begin{tabular}{|l|c|c|c|c|}
\multicolumn{1}{l}{ Zone I } & Zone II & Zone III & Zone IV \\
\hline Risque climatique (aridité + instabilité) & ++ & + & + & - \\
\hline Population (densités + croissance) & ++ & ++ & - & + \\
\hline Pression sur ressources (eau + bois + jachère + mitage) & +++ & + & + & - \\
\hline Soudure (greniers + aide + emprunt + prix + feuilles) & +++ & ++ & + & - \\
\hline Enclavement (géographique + culturel) & + & - & ++ & - \\
\hline
\end{tabular}

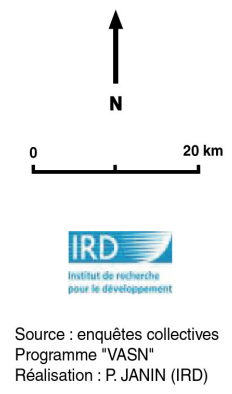

Cette première analyse de la vulnérabilité a été ensuite été déclinée à des échelles désagrégées, à l'aide d'enquêtes saisonnières ${ }^{5}$, afin d'évaluer la vulnérabilité réelle des ménages ruraux. Notre hypothèse centrale était que, même soumis à de fortes contraintes environnementales, avec des ressources monétaires et alimentaires réduites et un faible niveau d'information, un individu ou un ménage participe, par sa prise de décision, tantôt adéquate et opportune, tantôt retardée ou incomplète - dans la gestion combinée des ressources alimentaires (bétail et grenier) par exemple -, au renforcement de la vulnérabilité. Par ailleurs,

${ }^{5}$ L'échantillonnage a été effectué par tirage au sort après établissement de la liste complète des ménages, sur la base des cahiers administratifs villageois élaborés pour le recensement de 1998. 
la régulation exercée, dans le domaine de la mobilité socio-géographique, de l'accès aux facteurs de production ou à la ration alimentaire, par son caractère souvent inégalitaire et excluant, dévoile progressivement la part socialement construite de la vulnérabilité.

\begin{tabular}{|c|c|c|c|c|c|}
\hline \multicolumn{6}{|c|}{$\begin{array}{l}\text { Caractéristiques socio-démographiques des ménages }(n=579) \text { et des cours collectives }(n=180) \\
\text { selon les zones de risque apparent }\end{array}$} \\
\hline & $\begin{array}{c}\text { Zone I } \\
\text { (risque maxi) }\end{array}$ & Zone II & Zone III & $\begin{array}{c}\text { Zone IV } \\
\text { (risque mini) }\end{array}$ & $\begin{array}{c}\text { Comparaison } \\
\text { statistique }\end{array}$ \\
\hline Nombre de ménages par cour & 3,7 & 3,5 & 3,0 & 2,6 & $\begin{array}{l}\mathrm{F}=1,3 \\
\mathrm{P}=0,27\end{array}$ \\
\hline Nombre de sujets par cour & 36,5 & 30,5 & 28,2 & 26,4 & $\begin{array}{l}\mathrm{F}=1,0 \\
\mathrm{P}=0,41\end{array}$ \\
\hline $\begin{array}{ll}\text { Ethnie du CM : } & \text { - gourmantché } \\
(\text { en \%) } & \text { - peuhl } \\
& \text { - mossi } \\
\end{array}$ & $\begin{array}{l}88,5 \\
2,5 \\
9,0 \\
\end{array}$ & $\begin{array}{c}92,9 \\
6,6 \\
0,6 \\
\end{array}$ & $\begin{array}{c}76,0 \\
21,6 \\
2,4 \\
\end{array}$ & $\begin{array}{c}69,8 \\
27,9 \\
2,3 \\
\end{array}$ & $\begin{array}{l}\text { Chi2 }=76,7 \\
\mathrm{P}<0,0001\end{array}$ \\
\hline $\begin{array}{l}\% \text { de chefs de Ménage résidents } \\
\text { anciens (naissance ou }>50 \text { ans) }\end{array}$ & 92,5 & 74,9 & 71,7 & 85,9 & $\begin{array}{l}\text { Chi } 2=30,2 \\
\mathrm{P}<0,0001\end{array}$ \\
\hline $\begin{array}{ll}\text { Niveau patrimoine* }: & - \text { « riche » } \\
(\text { en \%) } & - \text { «moyen » } \\
& - \text { - pauvre » }\end{array}$ & $\begin{array}{l}38 \\
42 \\
20\end{array}$ & $\begin{array}{l}22 \\
37 \\
41\end{array}$ & $\begin{array}{l}40 \\
24 \\
36\end{array}$ & $\begin{array}{l}35 \\
36 \\
29\end{array}$ & $\begin{array}{l}\text { Chi } 2=27,4 \\
P<0,0001\end{array}$ \\
\hline$\%$ de ménages polygames & 47,5 & 53,6 & 44,0 & 60,5 & $\begin{array}{l}\text { Chi2 }=6,9 \\
\mathrm{P}=0,08\end{array}$ \\
\hline
\end{tabular}

Source : enquêtes IRD/Univ. Ouagadougou/IEDES-Paris I, novembre-décembre 2001. Sur 579 ménages et 180 cours. * Indice calculé à partir de la qualité du logement, des biens de consommation courante, de la possession de bovins.

Socio-demographic characteristics of households and collective dwelling units (or compounds) within apparent risk areas

Pour ce faire, nous avons opté pour des enquêtes très détaillées sur les dotations individuelles (parcelles, greniers, cheptel, revenus), leurs modes de gestion et la capacité réelle de maîtrise de ces ressources par leur responsable-gestionnaire en terme de droits. L'évaluation des disponibilités céréalières réelles, qu'il s'agisse des stocks familiaux et des achats céréaliers a fait l'objet d'une minutieuse reconstitution ${ }^{6}$, rendu souvent difficile par la pluralité des responsables (chef de famille, chef de ménage, épouse, autre actif masculin) mais également par la dispersion des parcelles cultivées et des lieux de stockage ${ }^{7}$. Elle a été réalisée en trois temps : après la récolte d'abord; en saison sèche ensuite, lorsque les réserves commencent à s'amenuisent ; en hivernage enfin, lorsque les déficits sont accentués.

Parallèlement, le rôle des régulations plus lointaines, marchande ${ }^{8}$ ou politique, n'a pas été ignoré même si son incidence sur les situations de vulnérabilité alimentaire des ménages reste statistiquement difficile à évaluer. En revanche, la contribution des réseaux marchands à la gestion du système alimentaire à très court terme apparaît cruciale en raison d'une bonne

\footnotetext{
${ }^{6}$ Chaque grenier numéroté a été dessiné sur un plan et a fait l'objet de mesures détaillées (capacité théorique, volume réel des céréales stockées en épis, conversion en kg-équivalents). Les différentes achats ont été répertoriés mois par mois sur des chnronogrammes.

${ }^{7}$ En effet, dans cet espace ouvert, où les terroirs sont imparfaitement fixés, les unités d'exploitation ne possèdent pas de véritable centralité.

${ }^{8}$ Une enquête auprès d'un échantillon de commerçants de céréales et de bétail opérant dans la province a été réalisée, sur huit marchés zonaux importants, associant un récit de vie succinct et une analyse détaillée des stratégies marchandes.
} 
réactivité ${ }^{9}$ A l'échelle des familles, le marché joue un rôle stratégique mais ambivalent en période de soudure alimentaire (Janin 2006) : fournisseur de denrées mais également facteur de précarisation. En dépit d'un enclavement géographique en saison des pluies, on observe en effet un phénomène croissant de marchandisation de certaines récoltes et du cheptel de l'exploitation. Se développe en outre, de plus en plus, un véritable "petit commerce » individuel (arachide, riz, maïs, sorgho et petits ruminants) destiné à générer des revenus indispensables à la couverture des besoins de base.

\section{La confrontation des dimensions}

Une des premières étapes de l'analyse a consisté à confronter les éléments géographiques de la vulnérabilité (de vulnérabilité apparente) avec les données de vulnérabilité réelle (tableau 2). On a alors observé que le niveau de risque apparent, essentiellement basé sur les contraintes environnementales au sens large, est loin de correspondre au niveau réel des déficits céréaliers mesurés. Ceci est également confirmé par les données recueillies lors de l'enquête 2002 suivante sur la soudure, de durée et d'intensité pourtant sensiblement inférieures. Là encore, la zone de risque maximal se singularise par une moindre vulnérabilité.

\begin{tabular}{cccccc}
\hline Tableau 2 \\
\hline Caractéristiques de la soudure 2001 dans les ménages selon les zones de risque \\
\hline & $\begin{array}{c}\text { Zone I } \\
\text { (risque maxi) }\end{array}$ & Zone II & Zone III & $\begin{array}{c}\text { Zone IV } \\
\text { (risque mini) }\end{array}$ & $\begin{array}{c}\text { Comparaison } \\
\text { statistique }\end{array}$ \\
\hline Nombre de mois d'anticipation & 3,0 & 1,8 & 2,8 & 2,3 & $\begin{array}{c}\mathrm{F}=3,9 \\
\mathrm{P}=0,009\end{array}$ \\
\hline $\begin{array}{c}\text { Achats céréaliers en \% des besoins } \\
\text { alimentaires annuels }\end{array}$ & 46,9 & 53,0 & 51,3 & 38,7 & $\begin{array}{c}\mathrm{F}=3,1 \\
\mathrm{P}=0,02\end{array}$ \\
\hline $\begin{array}{c}\text { Ajustement à la baisse } \\
\text { de la ration (en \%) }\end{array}$ & 52,2 & 58,7 & 70,4 & 54,8 & $\begin{array}{c}\mathrm{F}=5,4 \\
\mathrm{P}=0,008\end{array}$ \\
\hline $\begin{array}{c}\text { Nombre de jours de soudure } \\
\text { Yorouba / jour / équivalent-adulte }\end{array}$ & 175 & 210 & 180 & 157 & $\begin{array}{r}\mathrm{F}=7,9 \\
\mathrm{P}<0,0001\end{array}$ \\
\hline $\begin{array}{c}\text { Revenu annuel net moyen* } \\
\text { par ménage en FCFA }\end{array}$ & 226.373 & 98.379 & 142.061 & 155.364 & $\begin{array}{r}\mathrm{F}=7,1 \\
\mathrm{P}<0,0001\end{array}$ \\
\hline
\end{tabular}

Source : enquêtes IRD/Univ. Ouagadougou/IEDES-Paris I, novembre-décembre 2001. *La comparaison est effectuée sur la transformation logarithmique de la variable. Sur 579 ménages.

Basic characteristics of seasonal food shortage within apparent risk areas

L'insécurité alimentaire et la vulnérabilité réelles ne cadrent donc que très imparfaitement avec le diagnostic territorial préliminaire, même si elles s'en affranchissent pas totalement. Et encore ne s'agit-il que de données agrégées à l'échelle du ménage, qui ne prennent pas en compte les inégalités individuelles de ressources (argent, céréales, bétail) comme de droits, existants entre les individus, susceptibles d'aggraver les niveaux de vulnérabilité. Il paraît, de

\footnotetext{
${ }^{9}$ En 2000/2001, 51 commerçants interrogés ont commercialisé environ 71400 sacs de sorgho blanc et de mil contre 60700 sacs en 2001/2002 pour 39 d'entre eux. Ce volume représentait environ 10\% de la consommation globale annuelle des habitants de la province.
} 
ce fait, présomptueux et illusoire d'établir une cartographie régionale de la vulnérabilité, $a$ fortiori puisque les enquêtes ont porté sur un échantillon réduit de villages (30 sur 276).

Tout ceci suggère que, confrontés à la récurrence du phénomène de la soudure alimentaire, les ménages les plus exposés ont progressivement développé des stratégies permettant de gommer partiellement les aléas conjoncturels et ce malgré l'étroitesse des marges de manœuvre économique, agronomique et leur faible niveau d'information. Parmi les réponses les plus efficientes et compte tenu des efforts des ONG locales, on trouve certes un ensemble désormais classique «d'activités génératrices de revenu » telles que l'embouche, le maraîchage ou l'artisanat alimentaire. Mais compte tenu de la relative homogénéité des conditions de production, du niveau des ressources et des savoir-faire locaux, la vulnérabilité différenciée des ménages est davantage à rechercher dans la qualité de la prise de décision en situation d'incertitude et de forte contrainte qu'il s'agisse de l'anticipation temporelle des achats céréaliers (réalisés dès lors à moindre coût étant donné la volatilité des prix), du report d'utilisation des greniers familiaux ou des ajustements apportés à la ration alimentaire.

Par ailleurs, les différents indices nutritionnels de déficit énergétique et de maigreur aboutissent à la même hiérarchisation entre zones (Janin et Martin-Prével 2005). Vis-à-vis de l'état nutritionnel, les caractéristiques d'ordre socio-démographique (religion, statut, niveau d'éducation, ethnie) et économique (revenus) joueraient donc un rôle plus important que les conditions objectives de la soudure (importance du déficit céréalier) (Martin et al. 2004). Ces éléments se concrétisent par de meilleurs indices de diversité et de variété alimentaires en zone I qu'ailleurs.

\section{Les indicateurs avancés de suivi}

Cette première analyse met donc en évidence le hiatus existant entre la vulnérabilité alimentaire apparente, basée sur une cartographie thématique et la vulnérabilité réelle résultant de mesures plus individualisées. Elle montre aussi que les échelles d'observation spatiale, temporelle ou statistique, modifient quelque peu la nature des résultats. Un lien direct existe donc entre les choix conceptuels, méthodologiques ou logistiques d'une recherche, les présupposés idéologiques ou politiques (Janin et de Suremain 2005) et les objectifs visés (type d'intervention).

La variabilité spatio-temporelle des disponibilités comme de la capacité des ménages à faire face au phénomène de soudure plus ou moins longue et aggravée constitue, en outre, une difficulté supplémentaire pour un ciblage socio-géographique des interventions (aide alimentaire, micro-crédit).

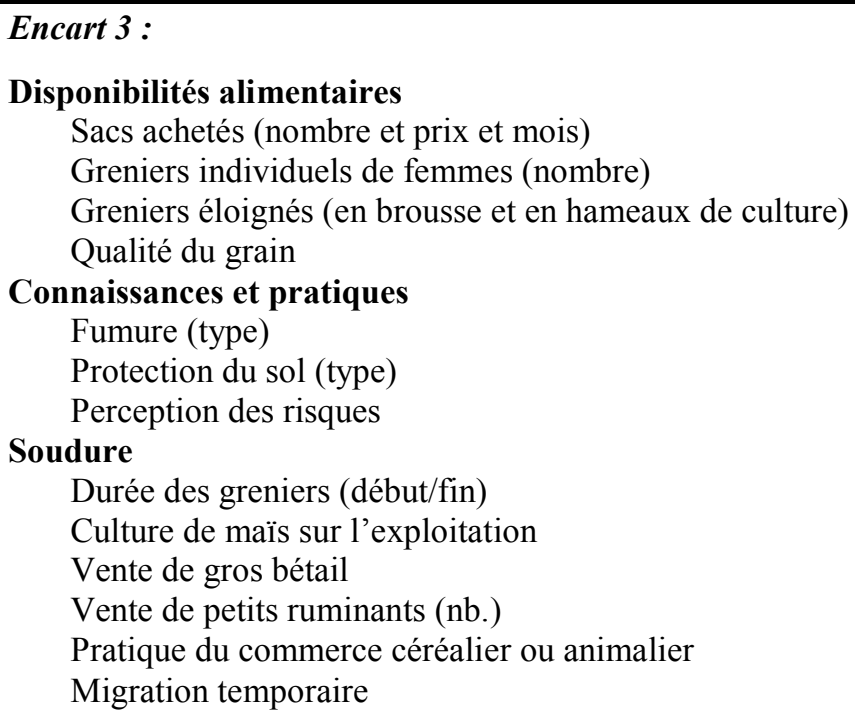


Consommation retardée des greniers

Reconstitution du cheptel

Indices de prix avancés

Consommation alimentaire

Evolution de la ration (saison sèche/travail agricole)

Consommation de feuilles de cueillette

Prise en charge d'autre ménage

Consommation à caractère « exceptionnel »

Aujourd'hui encore, le meilleur moyen de «passer la soudure » pour les ménages ruraux sahéliens réside encore dans une gestion combinée efficiente des stocks céréaliers et animaliers, avec un recours pérenne au marché, et dans l'élargissement de l'horizon d'anticipation, tout en jouant sur les marges de manœuvre (ajustement alimentaire ou démographique, amélioration de la fertilité et diversification d'activités (Ellis 1998 ; Barret et al. 2001) dont ils disposent.

Afin de répondre à d'éventuelles demandes de la part des décideurs, nous avons cherché à identifier des indicateurs, à la fois rapides à collecter et fiables, afin d'en faire un suivi, pour évaluer par avance, à la fois l'apparition de soudures plus longues ou plus aiguës que la moyenne et les éléments de gestion des parades qui se révèlent inadaptés, inefficaces ou tardifs.

Ont été considérés ici comme des indicateurs tous les repères, immédiatement observables ou résultant de déclarations (sur plusieurs années), de déséquilibre dans le temps et dans le paysage à l'échelle de la concession indiquant qu'une crise alimentaire plus grave que d'habitude peut intervenir et que les réponses des familles sont insuffisantes ou inappropriées. C'est cette sélection indicative que nous présentons ci-dessus. Nous pensons qu'elle constitue un point de départ pour un ciblage plus efficace des interventions.

Quels enseignements peut-on retenir de cette recherche multiscalaire et multi-acteurs sur la vulnérabilité alimentaire en milieu rural sahélien ?

Tout d'abord qu'une approche méthodologique pertinente s'enracine dans une connaissance intime des espaces et des sociétés à étudier. Elle ne peut résulter de la simple transposition de protocoles généralistes d'enquêtes déjà testés, établis et validés.

Ensuite, que les analyses statistiques permettent certes de caractériser le comportement des acteurs, voire de le modéliser mais que le suivi dynamique de la vulnérabilité et sa mesure réelle passent aussi par des analyses plus qualitatives en terme d'enjeux globaux, de perception temporelle du risque, de jeux d'acteurs.

Enfin, qu'un diagnostic géo-cartographique des risques apparents (sous forme d'indicateurs spatialisés) est une étape importante, sinon obligée, pour une meilleure prise en compte des "dires d'acteurs » mais que seule la mise au point d'analyseurs plus complexes, permet d'aborder la question du suivi dynamique de la vulnérabilité. Cette dernière se fonde, en effet, sur des inégalités individuelles masquées, en terme des ressources et de droits mobilisables, dans la mesure où elles se fondent sur des hiérarchies établies et bien souvent acceptées.

De fait, aborder la question alimentaire en milieu rural selon une démarche descriptive des déficits comme des surplus ou des réponses plus ou moins tactiques - nous semble un peu vain dès lors que la nature complexe des arbitrages sociaux (inégalités de droits d'accès et de gestion) n'est pas envisagée.

Pour un chercheur en sciences sociales, une telle recherche a constitué un apprentissage exigeant de l'incertitude dans son quotidien le plus banal et la confirmation que le pire n'est jamais certain, même en situation de grande précarité, compte tenu de la qualité du lien social. 


\section{Références}

ADGER W.N., (1999). "Social vulnerability to climate change and extremes in coastal Vietnam". World Development, vol. 27, $\mathrm{n}^{\circ}$ 2, p. 249-269.

BANQUE MONDIALE, (2001). «Aider les pauvres à gérer les risques ». In : Rapport mondial sur le dévloppement dans le monde 2000-2001, Banque Mondiale, Wahington, p. 157-188.

BARRET C.B., REARDON T., WEBB P., (2001). "Non-farm income diversification and household livelihood strategies in rural Africa : concepts, dynamics, and policy implications". Food Policy, vol. 26, p. 315-331.

BEBBINGTON A., (1999). "Capitals and capacities : a framework for analyzing peasant viability, rural livelihoods and poverty", World Development, vol. 27, n 12, p. 2021-2044.

Blein R., TRAORE K., Jeudy E., (2001). «Améliorer la capacité sahélienne d'anticipation et de gestion des crises alimentaires majeures ». Communication au Réseau de prévention des crises alimentaires (28-30 novembre 2001), Club du Sahel-OCDE-CILSS, 21 p.

BRIAND V., (2004). Vulnérabilité et insécurité alimentaire. Le cas des unités domestiques de Bouaké. Thèse de doctorat, Nanterre-Université Paris X, 451 p.

BURTON I., (1997). "Vulnerability and adaptative response in the context of climate and climate change". Climatic Change, vol. 36, n 1-2, p. 185-196.

CAMBreZY L., JANIN P., (2003). « Le risque alimentaire en Afrique ». In : VeYRET Y. éd. Les risques, Paris, Col. Dossiers des images économiques du monde (DIEM), SEDES, p. 88-103.

Chastanet M., (1991). "Crise et régulation en pays soninké (Sénégal) depuis le début du XIX ${ }^{\circ}$ siècle ». Paris, ORSTOM , Cahiers des Sciences Humaines, vol. 27, n 1-2, p. 131-145.

COURADE G., (1998). «Ajustement structurel et ouverture des marchés: moins de pénuries mais de nouveaux risques », Canadian Journal of Development Studies, vol. 14, p. 123-139.

COURADE G., et al., (2001). «Inégalités, vulnérabilité et résilience : les voies étroites d'un nouveau contrat social en Afrique subsaharienne ». In : WINTER G. coord. Inégalités et politiques publiques en Afrique. Pluralité des normes et jeux d'acteurs. Paris, Karthala, Coll. Economies et sociétés, p. 119-133.

COURADE G., (2001). «Paupérisation et inégalités d'accès aux ressources ». In : WINTER G. coord. Inégalités et politiques publiques en Afrique. Pluralité des normes et jeux d'acteurs. Paris, Karthala, Coll. Economies et sociétés, p. 25-39.

Delor F., and HUBERT M., (2000). "Revisiting the concept of vulnerability". Social Science and Medicine, vol. 50, p. $1557-1570$.

Dilley M. and BoudreaU T. E., (2001). "Coming to terms with vulnerability : a critique of the food security definition". Food Policy, vol. 26, p. 229-247.

DUPRE G., GUILlaUd D., (1999). « Entre incertitude et sécurité : les systèmes de production en Aribinda (Burkina Faso) ». In : CHAMPAUD J. et PONCET Y. éds. Les temps du Sahel, Paris, IRD, p. 109-130.

ELLIS F., (1998). "Household livelihood strategies and rural livelihood diversification". Journal of Development Studies, vol. $35, \mathrm{n}^{\circ} 1, \mathrm{p} .1-38$.

GADO B. A., (1993). Une histoire des famines au Sahel. Etude des grandes crises alimentaires (19-20 ${ }^{e}$ siècle). L'Harmattan, Paris, $201 \mathrm{p}$.

Gallais J., (1984). Hommes du Sahel. Paris, Flammarion, Coll. Géographes, 289 p.

GARAVINI M., (1997). La définition du concept de risque dans le cadre d'un système d'alerte précoce agrométéorologique. CILSS-OMM, $61 \mathrm{p}$.

HAMELIN A.-M., BEAUdRY M., HABICHT J.-P., (1998). «La vulnérabilité des ménages à l'insécurité ». In : La quête de la sécurité alimentaire au $21^{e}$ siècle, Revue canadienne d'études du développement, Université d'Ottawa, vol. 19, p. 278-306.

Heidhues F., Atsain A., Nyangito H., Padilla M., Ghersi G., Le Vallee J.-C., (2004). Development Strategies and Food and Nutrition Security in Africa. Wahington, IFPRI, $60 \mathrm{p}$.

IFPRI. Stratégie de l'IFPRI vers la sécurité alimentaire et nutritionnelle. Recherche sur les politiques alimentaires, reforcement des capacités, communication des politiques, Washington, avril 2003, 37 p.

JANIN P., (2006). "L'ambivalence du marché dans la sécurisation alimentaire au Sahel », Paris, AFD, Afrique contemporaine, Bruxelles, De Boeck Université, AFD, Afrique Contemporaine, Dossier Agricultures familiales en Afrique subsaharienne, $\mathrm{n}^{\circ}$ 217, 1/2006, pp. 91-105.

JANIN P., (2004). « La gestion spatio-temporelle de la soudure alimentaire dans le Sahel burkinabé ». Paris, PUF, Revue Tiers-Monde, $\mathrm{n}^{\circ}$ 180, p. 909-933.

JANIN P., (2001). «L'insécurité alimentaire rurale en Côte d'Ivoire : une réalité cachée, aggravée par la société et le marché ». Cahiers Agricultures, Paris, John Libbey Eurotext, vol. 10, n 4, p. 233-241.

JANIN P., MARTIN-PREVEL Y. (2006). « Des indicateurs à l'action : vulnérabilité alimentaire et états nutritionnels en milieu rural sahélien burkinabè », Ottawa, Revue canadienne d'Etudes Africaines, Vol. 40, n 3, 443-461.

JANIN P. et SUREMAIN CH.-E., 2005. - La question alimentaire en Afrique : risque et politisation, Paris, PUF, Revue Tiers-Monde, octobre-décembre 2005, $\mathrm{n}^{\circ} 184$, pp. 727-859. 
JASPERS S., SHOHAM J., (1999). "Targeting the vulnerable : a review of the necessity and feasability of targeting vulnerable households". Disasters, vol. 23, n 4, p. 359-372.

MAKKI S.S., TwEETEN L.G., MIRANDA M.J., (2001). "Storage-trade interactions under uncertainty. Implications for food security". Journal of Policy modeling, vol. 23, p. 127-140.

Marchal J.-Y., (1990). «En Afrique soudano-sahélienne: la course contre le temps ». In: EldiN M. et MILlEVILLE P. eds. Le risque en agriculture, Paris, ORSTOM, Col. A travers champs, p. 225-267.

MARTIN K.S., Rogers B.L., COOK J.T., JOSEPH H.M., (2004). "Social capital is associated with decreased hunger." Social Science and Medicine, vol. 58, p. 2645-2654.

Moseley W.G., (2001). "African evidence on the relation of poverty, time preference and the environment". Ecological Economics, vol. 38, p. 317-326.

MOSELEY W.G., LOGAN B.I., (2001). "Conceptualizing hunger dynamics : a critical examination of two early warning systems in Zimbabwe". Applied Geography, vol. 21, p. 223-248.

OuEDRAOGO F., (2001). Atlas de la province de la Gnagna. Essai de géographie des disparités spatiales de la vulnérabilité alimentaire, IRD, Ouagadougou, $152 \mathrm{p}$.

Raynaut C., dir. (1998). Sahels. Diversité et dynamiques des relations sociétés-nature. Paris, Karthala, Col. Hommes et sociétés, $430 \mathrm{p}$.

REARDON T., MATLON P., (1989). "Seasonal food insecurity and vulnerability in drought-affected regions of Burkina Faso". In : SAHN D. (1989). Seasonal variation in the Third World agriculture, John Hopkins University Press, Baltimore, p. 118-136.

RiBot J.C., MAgalhães A.R., PANAGIDES S.S., eds. (1996). Climate variability, climate change and social vulnerability in the semi-arid Tropics, Cambridge Press University, Cambridge, $175 \mathrm{p}$.

RESQUIERS-DESJARDINS D., (1995). «Impact des stabilités et gestion du risque et de l'incertitude alimentaire en Afrique Subsaharienne », In : HugON P. éd. (1995). L'Afrique des incertitudes, Paris, PuF, Col. Tiers-Monde, p. $221-238$

TURNER M.D., (2002). "Livestock Markets Dynamics and local Vulnerabilities in the Sahel", World Development Washington, Elsevier, vol. 30, n 4, p. 683-705.

WFP, (2001). Chronic Vulnerability to Food Insecurity in Kenya. A WFP Pilot Study for Improving Vulnerability Analysis, WFP, 36 p.

WFP. (2002). Vulnerability Analysis and Mapping Standard Analytical Framework. WFP, 11 p. 